\title{
The EWAS Catalog: a database of epigenome-wide as- sociation studies
}

Thomas Battram ${ }^{1,3^{*}}$, Paul Yousefi1,3, Gemma Crawford1,3, Claire Prince ${ }^{1,3}$, Mahsa Sheikhali Babaei1,3, Gemma Sharp ${ }^{1,3}$, Charlie Hatcher ${ }^{1,3}$, María Jesús Vega-Salas², Sahar Khodabakhsh², Oliver Whitehurst ${ }^{3}$, Ryan Langdon¹,3, Luke Mahoney ${ }^{3}$, Hannah R. Elliott1,3, Giulia Mancano1,3, Matthew A. Lee ${ }^{1,3}$, Sarah H. Watkins ${ }^{1,3}$, Abigail C. Lay ${ }^{4}$, Gibran Hemani1,3, Tom R. Gaunt ${ }^{1,3}$, Caroline L. Relton ${ }^{1,3}$, James R. Staley ${ }^{1,3+}$, Matthew Suderman 1,3+

${ }^{1} \mathrm{MRC}$ Integrative Epidemiology Unit, University of Bristol, Bristol BS8 1TH.

2 Centre for Exercise, Nutrition and Health Sciences, University of Bristol, Bristol BS8 1TH

${ }^{3}$ Bristol Medical School, University of Bristol, Bristol BS8 1TH

${ }^{4}$ Bristol Renal, Translational Health Sciences, Bristol Medical School, University of Bristol, Bristol, United Kingdom

${ }^{*}$ To whom correspondence should be addressed.

+Authors made equal contributions

\section{Summary}

Epigenome-wide association studies (EWAS) seek to quantify associations between traits/exposures and DNA methylation measured at thousands or millions of $\mathrm{CpG}$ sites across the genome. In recent years, the increase in availability of DNA methylation measures in population-based cohorts and case-control studies has resulted in a dramatic expansion of the number of EWAS being performed and published. To make this rich source of results more accessible, we have manually curated a database of $\mathrm{CpG}$-trait associations (with $\mathrm{p}<1 \times 10^{-4}$ ) from published EWAS, each assaying over 100,000 CpGs in at least 100 individuals. From 2021-01-29, The EWAS Catalog contained 1,045,303 associations from over 1000 EWAS. This includes 652,530 associations from 264 peer-reviewed publications. In addition, it also contains summary statistics for 392,773 associations from 428 EWAS, performed in data from the Avon Longitudinal Study of Parents and Children (ALSPAC) and the Gene Expression Omnibus (GEO). The database is accompanied by a web-based tool and R package, giving researchers the opportunity to quickly and easily query EWAS associations and gain insight into the molecular underpinnings of disease as well as the impact of traits and exposures on the DNA methylome.

The Ewas Catalog is available at: http://www.ewascatalog.org.

\section{Introduction}

EWAS assess associations between traits of interest and DNA methylation across the genome [1]-[3]. These associations may be used to gain mechanistic insights into disease and developmental processes or serve as molecular biomarkers in prediction applications [1]-[3]. Giving researchers easy access to the data will likely improve understanding of complex traits and may yield other translational benefits.

The EWAS Atlas has previously collated well-curated EWAS on traits in an online database and makes annotated $\mathrm{CpG}$ site-level results accessible via a website [4]. Other databases are available but are limited to certain diseases (e.g. MethHC [5]).

Ideally, a database of EWAS results will provide summary statistics, including effect estimates, standard errors, and p-values in an easily accessible manner, so that researchers can explore associations without having to retrieve the original article. For example, allowing comparison of effect estimates between studies or a look-up of specific associations to evaluate replication. Unfortunately, although EWAS Atlas does provide association p-values, it does not provide effect estimates and standard errors for download. For completeness, such a database should also where possible provide summary statistics for all potentially true associations beyond those passing conservative significance thresholds, but publications rarely report sub-threshold lists of associations. The contents of EWAS Atlas have todate been restricted to published associations. 
We therefore aimed to improve upon current databases to 1) provide all relevant summary statistics from a range of EWAS and 2) allow easy and programmatic access to results. To this end we have produced The EWAS Catalog, a manually curated database of currently published EWAS with additional data from 387 EWAS performed in ALSPAC [6], [7], and 41 EWAS performed with publicly available data from the GEO database (https://www.ncbi.nlm.nih.gov/geo/). The process and data inclusion are summarised in Supplementary Figure 1. The EWAS Catalog also enables users to upload results, allowing collection of results not necessarily reported in publications.

\section{Methods}

\subsection{Implementation}

The EWAS Catalog web app was built using the Django Python package (https://djangoproject.com). The data is stored in a combination of a MySQL database and fast random access files [8], and can be queried via the web (http://www.ewascatalog/org) or the R package (https://github.com/MRCIEU/ewascatalog-r).

\subsection{Overview of publication data extraction}

To identify publications, we perform periodic literature searches in PubMed using the terms: "epigenome-wide" OR "epigenome wide" OR "EWAS" OR "genome-wide AND methylation" OR "genome wide AND methylation".

Our criteria for inclusion of EWAS are as follows:

1. The analysis contains over 100 humans

2. The analysis contains over $100,000 \mathrm{CpG}$ sites

3. DNA methylation data is genome-wide (not a candidate gene study)

4. Results are not duplicated from a previous study

5. CpG-trait associations at $\mathrm{p}<1 \times 10^{-4}$ are reported

These criteria and the variables extracted are documented on the website (www.ewascatalog.org/documentation). Briefly, extracted variables include: the exposure variable, the outcome variable, the covariates, tissue, sample size, age, sex, reported ancestry or ethnicity, CpG IDs, effect estimates, standard errors, p-values. To unify representation of traits, they were mapped to Experimental Factor Ontology (EFO) terms, which were manually extracted from the European Bioinformatics Institute database (www.ebi.ac.uk/efo $)$.

\subsection{EWAS study data}

\subsubsection{Avon Longitudinal Study of Parents and Children (ALSPAC)}

EWAS were conducted for 387 continuous and binary traits (Supplementary Table 1) using DNA methylation measured in peripheral blood of ALSPAC mothers in middle age $(\mathrm{N}=940)$. The trait data were extracted from information collected at the same sampling point blood was drawn for DNA methylation assays. Quality control steps for the traits and information on the cohort are in the Supplementary Material.

\subsubsection{GEO datasets}

Full EWAS results were also estimated for studies that did not report complete summary statistics in their initial publication but where complete DNA methylation and trait of interest information were publicly available through the GEO database. We used the geograbi package (https://github.com/yousefi138/geograbi) to query GEO for experiments matching inclusion criteria (described above) and extract data for EWAS re-analysis. Details on the query and quality control can be found in the Supplementary Material. Both original published results and the full reanalysed GEO results have been included in The EWAS Catalog database. A list of all 41 traits with corresponding citations is provided in Supplementary Table 2. 
Details on the statistical analyses for EWAS performed specifically for the EWAS Catalog can be found in the Supplementary Material.

From 2021-01-29, The EWAS Catalog contained 1,045,303 associations from over 1000 EWAS.

\section{Results}

The database can be queried at www.ewascatalog.org or by using the R package "ewascatalog." The website provides a simple user interface with a search bar to explore the database as well as documentation on the catalog contents and how to cite its use (Figure 1). Basic queries may include a $\mathrm{CpG}$ identifier, gene symbol, genome region, trait, author name or PubMed ID. Query submission will then lead to an intermediate 'splash page' providing options for more specific queries. For example, a query for a specific trait would lead to a 'splash page' listing that trait, related traits, and all studies of that trait. Selecting one of these leads to a list of relevant EWAS associations, including CpG ID, trait, sample size, publication and association (effect size and p-value) (Supplementary Figure 2). This information, along with further details such as reported ancestry, outcome, exposure units and tissue analysed, are available for download as a tab-delimited text file. Alternatively, advanced queries are also supported wherein both a $\mathrm{CpG}$ identifier, gene symbol or genomic region are specified along with a trait, author name or PubMed ID. These queries are more specific and lead directly to a list of relevant EWAS associations.

Unlike other EWAS databases, we provide the option of downloading summary results for both the user's search and for the entire database. For details of how to query the database using the R package, see Supplementary Materials.

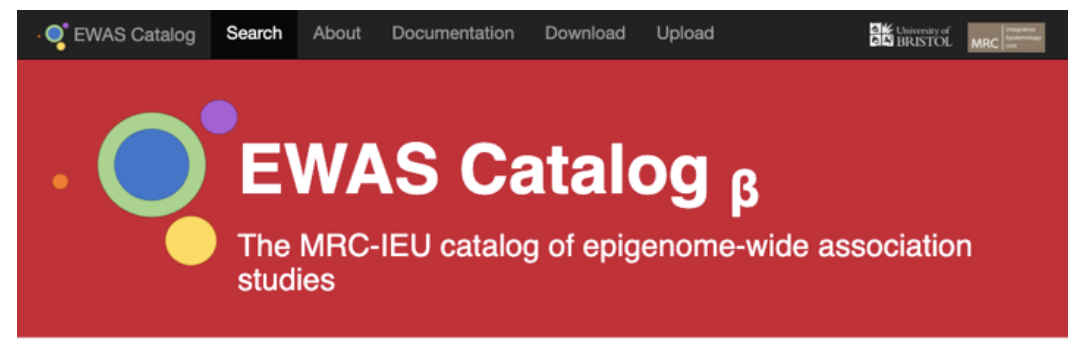

\section{Search}

Enter CpG, gene, region, trait, EFO term, author name or PMID

Examples: cg00029284, chr12:111731203, FTO, 6:15000000-25000000, body mass index, 27040690 .

+Advanced

Figure 1 The EWAS Catalog home page. From here users can search the database, view documentation and navigate to pages that allow for download of the full database and upload of user results. An example of results can be found in Supplementary Figure 2 .

\section{Discussion and future developments}

The EWAS Catalog provides a database of summary statistics from currently published EWAS and an additional 428 currently unpublished EWAS. This database has similar aims to the EWAS Atlas but has additional data sources, provides extra useful information and a user upload option. The EWAS Catalog team will continue to collate and upload newly published EWAS and perform additional EWAS on available datasets, whilst encouraging EWAS authors to upload their own summary data. We are currently working to incorporate additional functionality to allow users to systematically compare their own EWAS findings to EWAS already in the database. 


\section{Acknowledgements}

We are extremely grateful to all the families who took part in this study, the midwives for their help in recruiting them, and the whole ALSPAC team, which includes interviewers, computer and laboratory technicians, clerical workers, research scientists, volunteers, managers, receptionists and nurses.

\section{Funding}

This work was partly supported by a Wellcome Trust PhD studentship to TB (203746). This work was also supported by the BBSRC and ESRC (grant number ES/N000498/1) and by the UK Medical Research Council (MC_UU_00011/1, MC_UU_00011/4, MC_UU_12013/1, MC_UU_12013/2 and MC_UU_12013/4), which funds a Unit at the University of Bristol where all the authors work. The UK Medical Research Council and Wellcome (Grant ref: 217065/Z/19/Z) and the University of Bristol provide core support for ALSPAC. This publication is the work of the authors and TB, MS and JS will serve as guarantors for the contents of this paper. Methylation data in the ALSPAC cohort were generated as part of the UK BBSRC funded (BB/I025751/1 and BB/ I025263/1) Accessible Resource for Integrated Epigenomic Studies (ARIES) [http://www.ariesepigenomics.org.uk]. The phenotype collection was also in part funded by The British Heart Foundation (SP/07/008/24066), Roche Diagnostics and the National Institute for Health Research (NF-SI-0611-10196). A comprehensive list of grants funding is available on the ALSPAC website (http://www.bristol.ac.uk/alspac/external/documents/grant-acknowledgements.pdf)

Conflict of Interest: none declared.

\section{References}

[1] C. L. Relton and G. Davey Smith, "Epigenetic Epidemiology of Common Complex Disease: Prospects for Prediction, Prevention, and Treatment," PLoS Med., vol. 7, no. 10, p. e1000356, Oct. 2010.

[2] J. Mill and B. T. Heijmans, "From promises to practical strategies in epigenetic epidemiology," Nat. Rev. Genet., vol. 14, no. 8, pp. 585-594, 2013.

[3] V. K. Rakyan, T. A. Down, D. J. Balding, and S. Beck, "Epigenome-wide association studies for common human diseases," Nat. Rev. Genet., vol. 12, no. 8, pp. 529-541, Aug. 2011.

[4] M. Li et al., "EWAS Atlas: A curated knowledgebase of epigenome-wide association studies," Nucleic Acids Res., 2019.

[5] W. Y. Huang et al., "MethHC: A database of DNA methylation and gene expression in human cancer," Nucleic Acids Res., 2015.

[6] A. Fraser et al., "Cohort profile: The avon longitudinal study of parents and children: ALSPAC mothers cohort," Int. J. Epidemiol., 2013.

[7] A. Boyd et al., "Cohort profile: The 'Children of the 90s'-The index offspring of the avon longitudinal study of parents and children," Int. J. Epidemiol., vol. 42, no. 1, pp. 111-127, 2013.

[8] H. Li, "Tabix: Fast retrieval of sequence features from generic TAB-delimited files," Bioinformatics, 2011. 